\title{
Membrane patterns in eyes with choroidal neovascularization on optical coherence tomography angiography
}

\author{
Murat Karacorlu$^{1} \cdot$ Isil Sayman Muslubas ${ }^{1} \cdot$ Serra Arf $^{1} \cdot$ Mumin Hocaoglu $^{1} \cdot$ M. Giray Ersoz ${ }^{1}$ \\ Received: 13 September 2018 / Revised: 4 February 2019 / Accepted: 7 February 2019 / Published online: 1 April 2019 \\ (c) The Royal College of Ophthalmologists 2019
}

\begin{abstract}
Background To evaluate morphologic patterns of choroidal neovascular membranes using optical coherence tomography angiography (OCTA) in patients with treatment-naive, continuously treated, and previously treated exudative age-related macular degeneration (AMD).

Subjects We assessed retrospectively 184 eyes of 153 patients diagnosed with type 1, type 2, and mixed-type neovascularization associated with AMD. The type of neovascularization and clinical activity were assessed by clinical examination and spectral domain optical coherence tomography (SD-OCT). Morphological patterns of neovascular membranes were categorized using en face images on the AngioVue (Optovue) OCTA system.

Results The mean age of patients was $77.9 \pm 8.6$ years (range, 52-96 years). The most frequently identified type of membrane morphology was well-defined in the treatment-naive group (69\% of the eyes) and in eyes receiving ongoing antiVEGF treatments (77\% of the eyes). Long-filamentous morphology was the most frequent type in the previously treated group (53\%), in which only 33\% had a well-defined membrane. All clinically active cases had a well-defined pattern, such as a medusa or sea-fan shaped pattern, or an ill-defined pattern, and none had a long-filamentous neovascular network. Almost half of the clinically inactive cases $(47 \%)$ had well- or ill-defined, identifiable membrane morphology on OCTA. A longfilamentous membrane pattern, which was consistent with chronicity of lesion, was seen only in eyes with inactive neovascularization.

Conclusions The membrane morphology on OCTA was not associated with clinical activity, except that the presence of long dilated filamentous linear vessels was associated with chronicity and lesion inactivity.
\end{abstract}

\section{Introduction}

Age-related macular degeneration (AMD) is the leading cause of irreversible central visual loss in the elderly population. Clinically, the disease is divided into the dry (nonexudative) and wet (exudative, neovascular) forms. Type 1 neovascularization, which is the most common subtype of wet AMD, originates from the choroid and lies between the retinal pigment epithelium (RPE) and Bruch membrane. Type 2 neovascularization, which is the less common form of wet AMD, passes through the RPE and is located between the RPE and sensory retina. In mixed-type neovascularization (type 1 and type 2), the lesion is below

\footnotetext{
Murat Karacorlu

mkaracorlu@gmail.com

1 Istanbul Retina Institute, Istanbul, Turkey
}

and above the RPE. Type 3 neovascularization, known as retinal angiomatous proliferation, involves deep retina, and when it proliferates under the sensory retina and RPE, may also involve choroid [1-4].

For many years, dye-based angiography and structural optical coherence tomography (OCT) played a key role in the accurate diagnosis and classification of neovascularization secondary to AMD [5]. Characteristic leakage patterns on fluorescein angiography (FA) help classify the type and indocyanine green angiography (ICGA) improves the ability to detect neovascular membrane, especially in patients with type 1 and type 3 neovascularization. However, dyebased angiography is an invasive procedure with associated risk of adverse events. Spectral domain OCT (SD-OCT) is widely used as a noninvasive, rapid, and safe imaging method for assessing the location of the neovascular membrane and disease activity. However, apart from FA and ICGA, evaluation is mainly based on indirect findings such as hyperreflectivity above the RPE or sub-RPE, or the 
presence of fluid accumulation, rather than an analysis of neovascular membrane directly [5-7].

Optical coherence tomography angiography (OCTA) is a novel imaging modality that allows noninvasive and rapid visualization of vascular plexuses at different depths, from the internal limiting membrane up to the choroid, by using the motion contrast between rapidly repeated OCT B-scans $[6,8,9]$. In the present study we evaluated morphologic patterns of neovascular membranes using this new imaging technology in patients with treatment-naive, continuously treated, or previously treated exudative AMD.

\section{Subjects and methods}

Patients with neovascularization secondary to AMD who had been evaluated in Istanbul Retina Institute between March 2017 and February 2018 were evaluated retrospectively, and 184 eligible eyes of 153 patients were assessed in this study. Written informed consent was obtained prior to the diagnostic and therapeutic procedures. The study protocol was approved by the Institutional Review Board of Şişli Memorial Hospital, Istanbul. The study adhered to the tenets of the Declaration of Helsinki.

Patients diagnosed with type 1, type 2, and mixed-type neovascularization associated with AMD, and in whom the membrane could be identified on OCTA were included in the study. The initial diagnosis of type and clinical activity were based on clinical examination and spectral domain OCT (SD-OCT) assessment. Fluorescein angiography and ICGA were not routinely performed, except when OCT or OCT angiography failed to confirm the diagnosis. If fundus examination revealed retinal edema, hemorrhage, lipid exudates, or RPE detachments, further examination by OCT was required. On SD-OCT, presence of subretinal hyperreflective material or pigment epithelial detachments with intraretinal, subretinal, or sub-RPE fluid were considered signs of active neovascularization. Inactive neovascularization was defined as a history of active neovascularization treated with anti-VEGF at least 6 months before the examination, and no signs of activity, either by clinical examination or by SD-OCT at the time of OCTA acquisition. As, all patients with inactive neovascularization had long follow-up in our clinic, the initial subtype of neovascularization could be established on the basis of their previous OCT, with or without fluorescein angiography and ICGA images. Eyes with a history of prior photodynamic therapy, myopic choroidal neovascularization, central serous chorioretinopathy, polypoidal choroidal vasculopathy, or any evidence of other retinal disorders were excluded. As type 3 neovascularization initially may not involve choroid, and membrane morphology was evaluated from OCT angiography of a choroidal slab with or without the outer retinal slab, eyes with any evidence of type 3 neovascularization were not included. Images with signal strength index $<40$ were considered poor-quality images and excluded from the study.

All participants received a comprehensive ophthalmic examination, including measurement of best corrected visual acuity (BCVA) by the Early Treatment Diabetic Retinopathy Study chart, refraction and intraocular pressure measured by applanation tonometer, and detailed anterior segment and fundus examination by using slitlamp biomicroscopy with a 90 diopter noncontact lens. The Spectralis OCT system (Heidelberg Engineering, Heidelberg, Germany) was used for fundus autofluorescence, macular scans, choroidal imaging, digital fluorescein angiography, and ICGA. OCTA images were obtained by using the AngioVue (Optovue) OCTA system on a commercially avaliable SD-OCT device (RTVue XR Avanti; Optovue, Fremont, CA, USA). The OCTA images were evaluated using split-spectrum amplitude-decorrelation angiography (SSADA) and an algorithm to visualize retinal and choroidal vasculature in a $6 \times 6 \mathrm{~mm}$ volume of the central part of the retina. The retina was scanned in both horizontal and vertical axes, with correction for movements of the eyes. Automatic segmentation of the retinal layers was performed by the viewing software and was used to generate en face projection images of the lesion. In the case of segmentation errors, the segmentation lines were manually adjusted to evaluate the neovascular lesion adequately.

On the en face images, the morphological patterns of the neovascular membranes were categorized by using previously described methods $[6,8,9]$. The membrane was identified as well-defined if the lesion had a well-defined shape (the vessels branched in all directions from the center of the lesion (medusa) or $>90 \%$ of the membrane radiated from one side of the lesion (sea-fan)) with presence of numerous tiny branching capillaries from the center to the periphery with or without arteriovenous anastomotic loops, or a peripheral arcade of small anastomotic and looping vessels, or was identified as long filamentous if the membrane had a dead-tree or prunedvascular-tree pattern with long dilated filamentous linear vessels without branching small-capillary networks. Membranes lacking such distinct membrane morphology but with branching small-capillary networks with or without arteriovenous anastomotic loops and the peripheral arcade of small anastomotic and looping vessels were determined to be ill-defined (Fig. 1).

The cohort of eyes was divided into three groups: treatment-naive eyes with an active neovascular membrane, eyes receiving ongoing anti-VEGF treatments for active neovascular membrane, and eyes with an inactive neovascular membrane at the time of evaluation. 

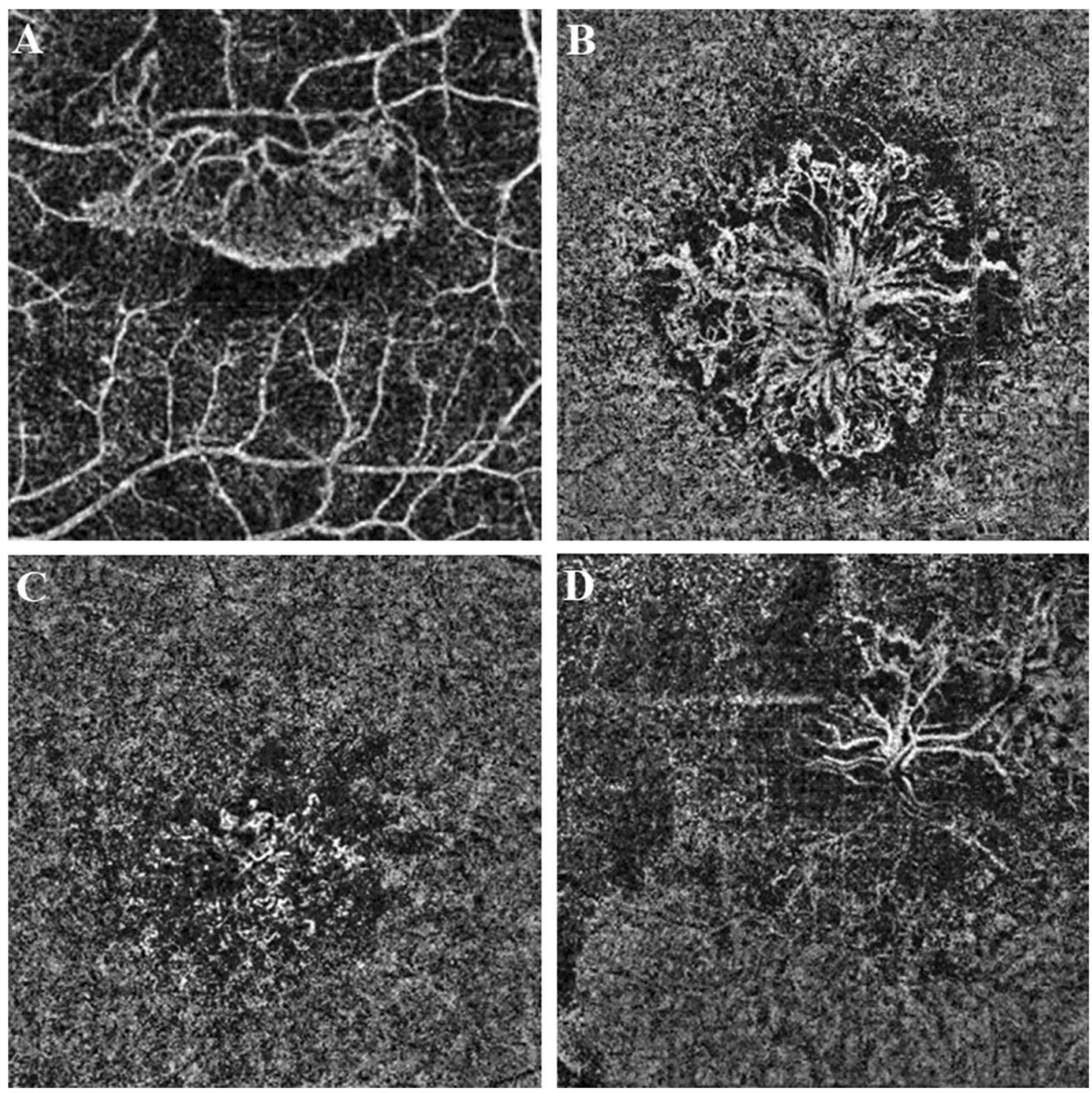

Fig. 1 The morphological patterns of the neovascular membranes on the optical coherence tomography angiography en face projection image. The membrane was identified as sea-fan if $>90 \%$ of the membrane radiated from one side of the lesion with presence of numerous tiny branching capillaries from the center to the periphery with or without arteriovenous anastomotic loops, or a peripheral arcade of small anastomotic and looping vessels (a). The membrane was identified as medusa if the vessels branched in all directions from the center of the lesion with presence of numerous tiny branching capillaries from the center to the periphery with or without

Demographic and clinical data were collected from the medical chart and data for all 184 eyes were used for statistical analysis. Descriptive statistical methods (mean, standard deviation) were used for characteristics such as age, sex, and duration of disease. Measurement values for the groups were compared by using one-way analysis of variance and the Pearson chi-square test. Statistical analyses used SPSS Version 20.0 (SPSS Inc, Chicago, IL, USA). $P<0.05$ was considered statistically significant.

arteriovenous anastomotic loops, or a peripheral arcade of small anastomotic and looping vessels (b). Membranes lacking such distinct membrane morphology but with branching small-capillary networks with or without arteriovenous anastomotic loops and the peripheral arcade of small anastomotic and looping vessels were determined to be ill-defined (c). The membrane was identified as long filamentous if the membrane had a dead-tree or pruned-vascular-tree pattern with long dilated filamentous linear vessels without branching small-capillary networks $(\mathbf{d})$

\section{Results}

One-hundred eighty-four eyes of 153 patients with neovascular AMD evaluated by OCTA were included in the study. One hundred fifty-three (83\%) eyes had type 1, 19 (10\%) eyes had type 2, and 12 (7\%) eyes had mixed type.

The mean age of patients was $77.9 \pm 8.6$ years (range, 52-96 years). Of the 135 eyes with active neovascularization, 39 were naive to anti-VEGF therapy, and 96 were 
Table 1 Baseline demographic and clinical characteristics

\begin{tabular}{|c|c|c|c|c|}
\hline Characteristics & $\begin{array}{l}\text { Treatment- } \\
\text { naive } \\
(n=39)\end{array}$ & $\begin{array}{l}\text { Continuously } \\
\text { treated }^{\text {a }} \\
(n=96)\end{array}$ & $\begin{array}{l}\text { Previously } \\
\text { treated }^{\text {b }} \\
(n=49)\end{array}$ & $P$ \\
\hline $\begin{array}{l}\text { Age }^{c} \text { (years) } \\
\text { (range) }\end{array}$ & $\begin{array}{l}76.5 \pm 9.6 \\
(52 \text { to } 96)\end{array}$ & $\begin{array}{l}78.6 \pm 8.1 \\
(55 \text { to } 93)\end{array}$ & $\begin{array}{l}78.2 \pm 8.6 \\
(57 \text { to } 93)\end{array}$ & 0.33 \\
\hline Male sex $(\%)$ & $17(32.7)$ & $44(37)$ & $25(42.2)$ & $0.57^{\mathrm{d}}$ \\
\hline $\begin{array}{l}\text { Mean disease } \\
\text { duration }^{\mathrm{e}} \\
\text { (months) }\end{array}$ & $1.5 \pm 1.2$ & $41.6 \pm 33.8$ & $52.1 \pm 39.9$ & $<0.01$ \\
\hline (range) & (1 to 6$)$ & (3 to 120 ) & (12 to 240 ) & \\
\hline $\begin{array}{l}\text { Mean number } \\
\text { of injections }{ }^{\mathrm{f}}\end{array}$ & 0 & $16.1 \pm 13$ & $10.4 \pm 5.8$ & $<0.01$ \\
\hline (range) & & (2 to 58$)$ & (3 to 28 ) & \\
\hline $\begin{array}{l}\text { Treatment } \\
\text { regimen }\end{array}$ & & & & $<0.01 \S$ \\
\hline $\mathrm{TE}(\%)$ & - & $83(86.4)$ & $33(67.3)$ & \\
\hline PRN (\%) & & 13 (13.6) & $16(32.7)$ & \\
\hline
\end{tabular}

Data, except percentages, are mean \pm standard deviation (range)

$T E$ treat and extend, $P R N$ pro re nata

${ }^{\text {a }}$ Patients who were receiving ongoing anti-VEGF treatment

${ }^{b}$ Patients who had a history of active neovascularization treated with anti-VEGF at least 6 months prior and no signs of activity at the time of evaluation

${ }^{\mathrm{c}}$ Treatment-naive vs. continuously treated $P=0.30$; Treatment-naive vs. previously treated $P=0.58$; Continuously treated vs. previously treated $P=0.93$, Tukey post-hoc test

${ }^{\mathrm{d}}$ Pearson chi-square test

'Treatment-naive vs. continuously treated $P<0.01$; Treatment-naive vs. previously treated $P<0.01$; Continuously treated vs. previously treated $P=0.09$, Tukey post-hoc test

${ }^{\mathrm{f}}$ Treatment-naive vs. continuously treated $P<0.01$; Treatment-naive vs. previously treated $P<0.01$; Continuously treated vs. previously treated $P=0.001$, Tukey post-hoc test

receiving ongoing anti-VEGF therapy (mean 16.1 \pm 13 injections during a mean $41.6 \pm 33.8$ months before the baseline examination). Forty-nine eyes with inactive neovascularization had received a mean of $10.4 \pm 5.8$ antiVEGF injections during a mean period $52.1 \pm 39.9$ months, at least 6 months before OCTA evaluation. There was a statistically significant difference in the mean duration of disease, mean number of injections and treatment regimens among groups (all, $P<0.01)$ (Table 1).

Of the 184 lesions, 117 (64\%) were well-defined (68 (37\%) medusa and 49 (27\%) sea-fan), which was the most common type of membrane. Twenty-six (14\%) lesions were identified as long filamentous, and $41(22 \%)$ had ill-defined morphology.

The most frequently identified membrane morphology was well-defined in treatment-naive (in 69\%) and in eyes receiving ongoing anti-VEGF treatments (in 77\%), but long-filamentous morphology was the most frequent type in the previously treated group (53\%), which had a welldefined membrane rate of only $33 \%$. There was a statistically significant difference in membrane morphology among groups $(P<0.001)$.

Ill-defined membrane morphology was detected in $31 \%$ of the treatment-naive eyes, $23 \%$ of eyes with ongoing treatment, and $14 \%$ of eyes with inactive neovascularization. There was no statistically significant difference in the presence of ill-defined membrane morphology among groups $(P=0.18)$

Among the 153 eyes with type 1 neovascularization, 90 (59\%) had well-defined, 41 (27\%) had ill-defined, and 22 (14\%) had long-filamentous membrane morphology. All eyes with type 2 and mixed-type neovascularization had distinct membrane morphology (well-defined or long filamentous). A long-filamentous membrane pattern was seen only in eyes with inactive neovascularization, and the presence of long-filamentous membrane morphology was associated with a significantly longer duration of disease (mean 52.1 months).

The morphological patterns of the neovascular membranes were demonstrated in detail in Figs. 2, 3 and 4.

\section{Discussion}

Optical coherence tomography angiography is a novel imaging modality for noninvasive qualitative and quantitative evaluation of neovascular lesions [9]. Many qualitative features of choroidal neovascularization have been assessed previously. Kuehlewein et al. [8] identified two distinct type 1 morphologies: the medusa and the sea-fan patterns; and indistinct morphology, with no obvious pattern or lack of detail. Sulzbacher et al. [10] suggested a new classification for wet AMD, based on OCTA findings: dense-net configuration (fine vessels with dense capillary branching), loose-net configuration (well-defined vessel with a low branching index), and an unidentifiable pattern (no neovascular vessels detectable) pattern. Miere et al. [11] also evaluated the neovascular pattern in a fibrotic scar; three patterns of vascular network could be distinguished, described as pruned-vascular-tree, tangled network, and/or vascular loop. In the present study, the membranes were identified as well-defined (medusa or sea-fan), long-filamentous, or ill-defined.

In exudative AMD, determination of vascular patterns of a neovascular lesion and its relation to clinical activity is essential. Correlation of clinical activity and the absence or presence of neovascularization on OCTA have been evaluated in previously [6, 12, 13]. Liang et al. [13] evaluated 25 eyes with clinically active and 20 eyes with clinically inactive AMD. The appearance of choroidal neovascularization was classified as well circumscribed if it appeared as a discrete 

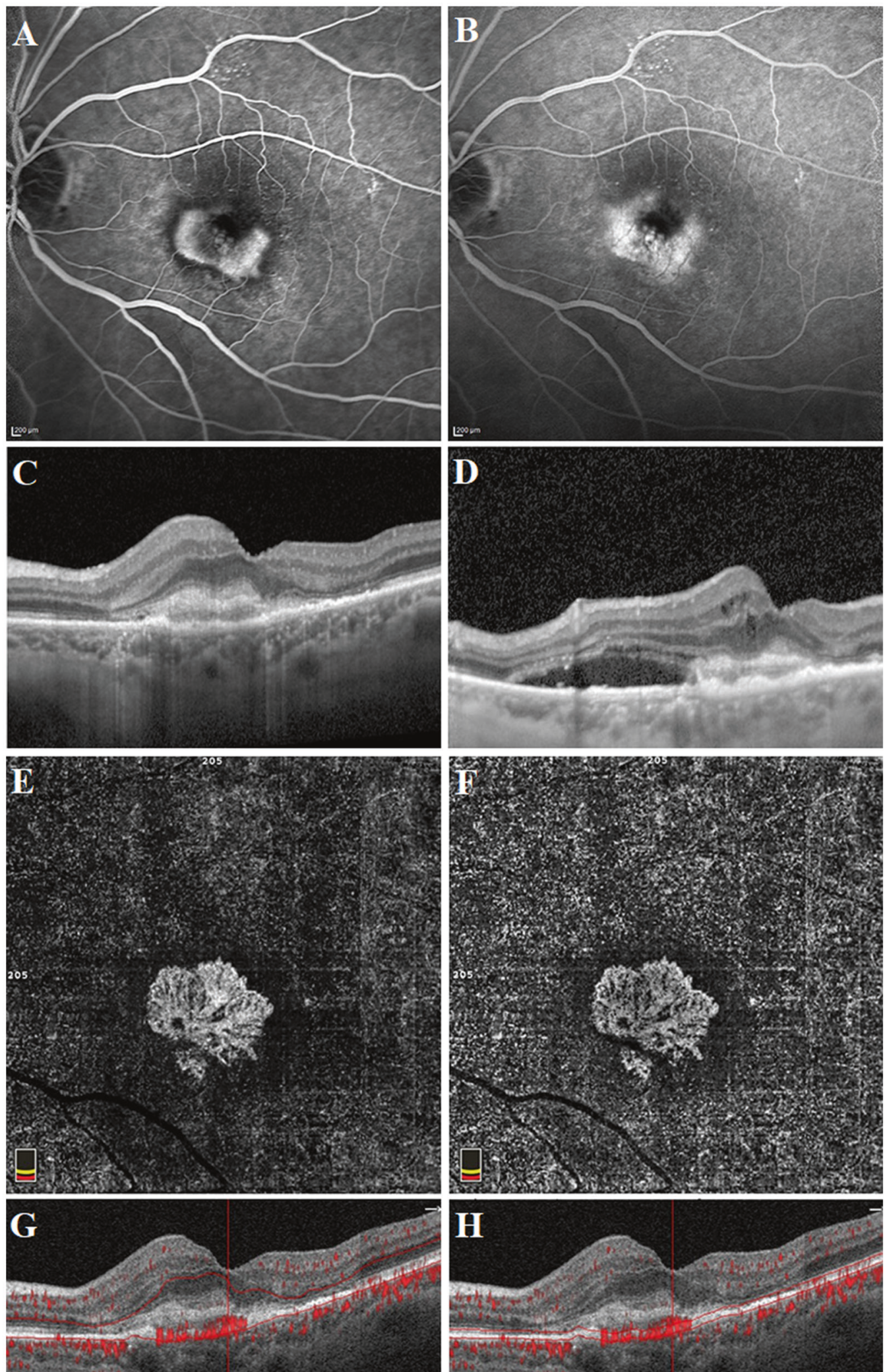

lesion composed of sea-fan shaped vessels or poorly circumscribed if it appeared as long-filamentous vessels without smaller vessels filling the spaces in between. Of those that

were clinically active, 22 had visible neovascularization on OCTA, and of those, 11 (50\%) had a sea-fan pattern and 11 (50\%) had long-filamentous vessels. Of the 20 eyes that were 
4 Fig. 2 Well-defined lesion with a sea-fan pattern in a treatment-naive patient with active type 2 neovascularization. Fluorescein angiography image demonstrating a hyperfluorescent area representing the neovascular complex during early phase (a) and concomitant retinal leakage delineating the retinal leakage area during late phase (b). Spectral-domain optical coherence tomography horizontal line scan indicated the neovascular complex above the retinal pigment epithelium (c). The adjacent subretinal fluid leakage was demonstrated on the spectral -domain optical coherence tomography vertical line scan (d). Optical coherence tomography angiography en face projection image of the neovascular complex with vessels radiating from 1 side of the lesion (sea-fan). The neovascular complex could be demonstrated, both in the outer retina $(\mathbf{e}, \mathbf{g})$ and choroid $(\mathbf{f}, \mathbf{h})$ slabs

clinically inactive, six had visible neovascularization, one $(17 \%)$ had a sea-fan pattern, and five (83\%) had longfilamentous vessels. It was reported that well-defined sea-fan vessels tended to be in the clinically active group, whereas poorly defined filamentous vessels could be associated with either active or inactive disease. Al-Sheikh et al. [12] also evaluated biomarkers of neovascular activity in AMD using OCTA and reported no correlation between the morphologic appearance of the membrane and clinical activity, similar to the study by Liang et al. [13].

In the study of Coscas et al. [6], patients with dead-tree appearance membrane morphology classified as patients "not requiring" treatment. Mierre et al. [11], evaluated imaging features of subretinal fibrosis secondary to exudative age-related macular degeneration on OCTA and they reported that the pruned-vascular tree was observed in $59.3 \%$ of the eyes with evidence of subretinal fibrosis that showed no exudative signs on SD-OCT in the last 6 moths. Also, in the study of Xu et al. [16], lesions were graded as having an overall immature, mature, or hyper-mature (deadtree appearance with long dilated filamentous straight vessels) vascular pattern. Of these morphologic biomarkers, the presence of an immature lesion and mature dilated core vessels were significantly associated with $\mathrm{CNV}$ growth. Lesions with dead-tree configuration were not significantly correlated with higher rates of $\mathrm{CNV}$ doubling, modest growth, or shrinkage. According to literature [6, 11, 16], dead-tree patterns and long-filamentous linear vessels have been associated with chronicity and inactivity.

In the present study, all cases found to be clinically active had a well-defined membrane morphology (such as medusa or sea-fan) or ill-defined morphology (membranes without such distinct morphology but with a branching small-capillary network with or without arteriovenous anastomotic loops and the peripheral arcade of small anastomotic and looping vessels), and none of the eyes had a long-filamentous neovascular network. Almost half (47\%) of the clinically inactive cases also had well- or ill-defined membrane morphology on OCTA. A long-filamentous membrane, which was consistent with the chronicity of the lesion, was seen only in eyes with inactive neovascularization. It could be concluded that appearance of a dead-tree or pruned-vascular-tree pattern with long dilated filamentous linear vessels on OCTA was associated with chronicity and inactivity of disease. However, the presence of vessels with a branching small-capillary network did not always mean clinically active disease. According to findings of our study, OCTA-based morphologic biomarkers are not always suitable as prognostic biomarkers for the course of the disease and subsequently as a guide for treatment decisions.

With anti-VEGF treatment, reduced intraocular VEGF levels cause closure of peripheral small capillaries and increased flow velocity, leading to dilation and maturation of vessels at the interior, a process that has been termed arteriogenesis, characterized by the appearance of largediameter vessels, loss of thin capillaries, and prominent anastomosis of vessels [14]. After the injection, pruning of smaller vessels is seen after $24 \mathrm{~h}$, with reduction of thinner anastomoses and loss of thinner capillaries. This pruning increases for 6 to 12 days. A reopening or new sprouting of the vessels is seen from 20 days to 40 days after each injection. The new vessels' proliferation and/or recanalization reappears. Pruning of the small peripheral branches seems to strengthen the trunk and cause an increased flow in the trunk [14-17]. Besides choroidal neo-arterialization, less exudative signal after treatment with anti-VEGF may allow better visualization of the neovascular membrane networks on OCTA. In the present study, we could not evaluate the vascular pattern changes with antiangiogenic therapy. We demonstrated ill-defined membrane morphology in 12/39 (31\%) treatment-naive eyes with an active neovascular membrane, in 22/96 (23\%) eyes receiving ongoing anti-VEGF treatments for active neovascular membrane, and in 7/49 (14\%) eyes with an inactive neovascular membrane. However, there was no statistically significant difference in the presence of ill-defined membrane morphology among groups, but it could be concluded that the rate of ill-defined membrane morphology was lower in eyes treated with anti-VEGF than in treatment-naive eyes, owing to better imaging of the neovascular membrane network after treatment.

Iafe et al. [18] reported that OCTA facilitated the morphological classification of type 1 lesions, including features characteristic of early, mature, and fibrotic lesions. Kuehlewein et al. [8] evaluated 33 eyes of patients with active or chronic type 1 neovascularization, and $18(55 \%)$ of the lesions were classified as medusa, $7(21 \%)$ as sea-fan, and $8(24 \%)$ as indistinct. There were no significant correlations by OCTA analysis between morphological patterns of neovascularization and the RPE detachment subtype nor with the number of intravitreal anti-VEGF injections received before imaging. Lupidi et al. [9] concluded that medusa or sea-fan shapes represented independent patterns, not passing through a sequential stage. In the present study, we could well identify 

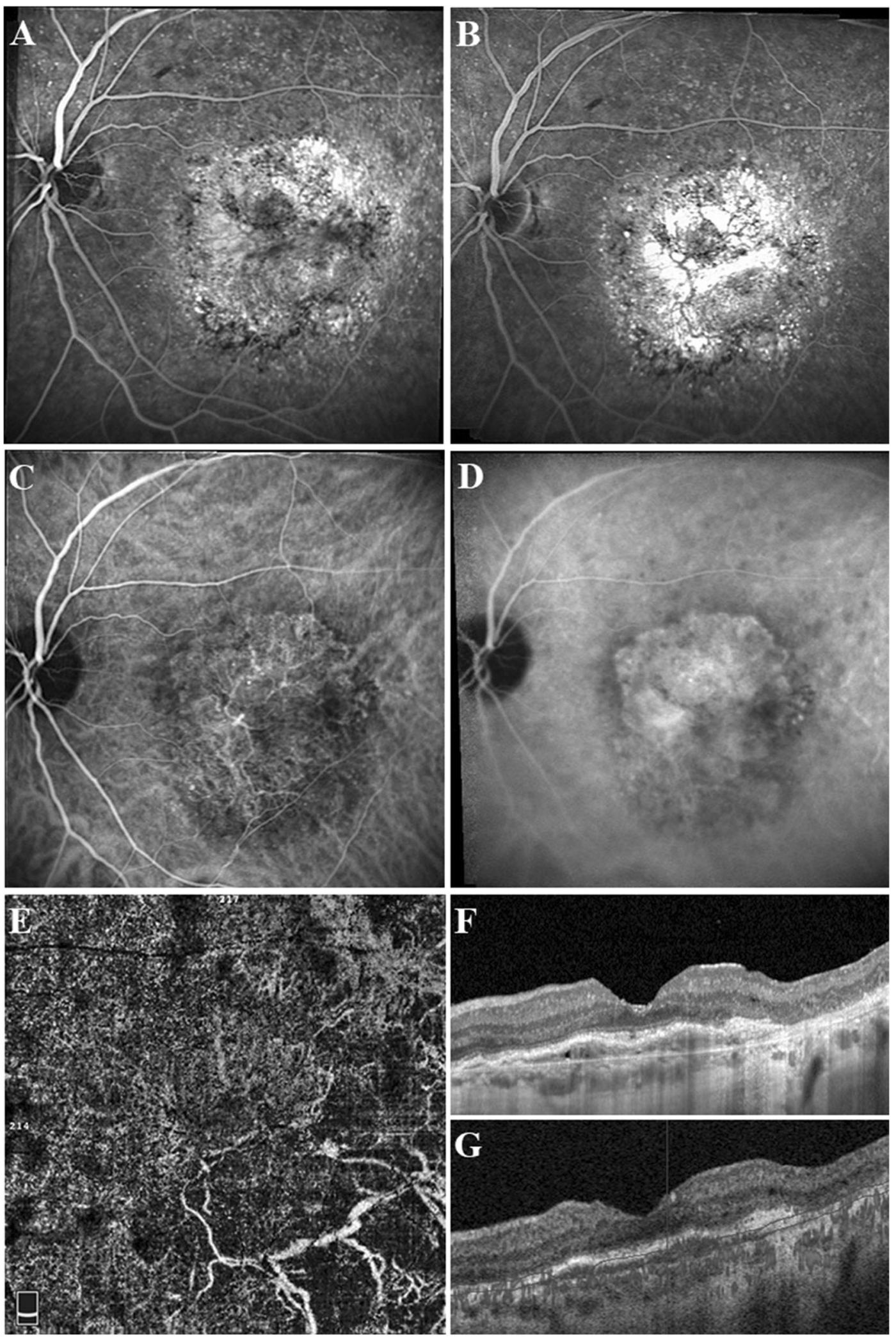

the membrane morphology on the OCTA en face projection of the choriocapillaris in eyes with active or inactive type 1 neovascularization, as medusa in 37\% (57/153), as sea-fan in
$22 \%$ (33/153), and as ill-defined in 27\% (41/153). A longfilamentous-vessel appearance was seen only in 22 eyes (14\%) with inactive type 1 neovascularization. 
Fig. 3 Long-filamentous vessels in a 80-year-old female patient who had a history of active type 1 neovascularization treated with eight anti-VEGF injections 5 years before the optical coherence tomography angiography acquisition. At the presentation, fluorescein angiography image demonstrated an ill-defined hyperfluorescent area during early phase (a) and late phase (b). Indocyanine green angiography image revealed the neovascular complex during early (c) and late phase (d). Optical coherence tomography angiography en face projection image of long dilated filamentous linear vessels without branching smallcapillary networks at the time of study evaluation (e). Spectral-domain optical coherence tomography horizontal line scan indicated the fibrovascular pigment epithelium detachment, but no subretinal or intraretinal fluid (f). Cross-sectional optical coherence tomography angiography revealed abnormal flow signal beneath the retinal pigment epithelium (g)

It is known that type 2 neovascularization demonstrates a demarcation from surrounding vasculature and can be identified on the mid-choroid slab to the outer retina [19, 20]. Ameen et al. [21] reported that type 2 lesions could be detected by OCTA, presenting as a hyperflow lesion in the outer retina, with a glomerulus or medusa shape in all cases. We could also demonstrate the neovascular complex, both in the choroid and outer retina slabs, in all cases with type 2 neovascularization, similar to previous reports. Most lesions were identified as sea-fan shapes (58\%).

In AMD patients with mixed-type neovascularization, OCTA is also an alternative method of identifying neovascularization [22]. Vessel morphology can be presented on the en face images both below and above the RPE, corresponding to different types of leakage observed on conventional angiography. In the present study, the distribution of the membrane morphology was: medusa in six eyes (50\%), sea-fan in five eyes (42\%), and long filamentous in one eye (8\%) Also, all lesions could be demonstrated both in the choroid and outer retina slabs.

In conclusion, OCTA enabled accurate information about morphological patterns of the neovascular membrane in patients with choroidal neovascularization. Well or ill-defined vessel patterns could be seen in eyes with clinically active or inactive choroidal neovascularization, but a long-filamentous-vessel pattern was seen only in eyes with inactive neovascularization. Although filamentous linear vessels on OCTA are associated with
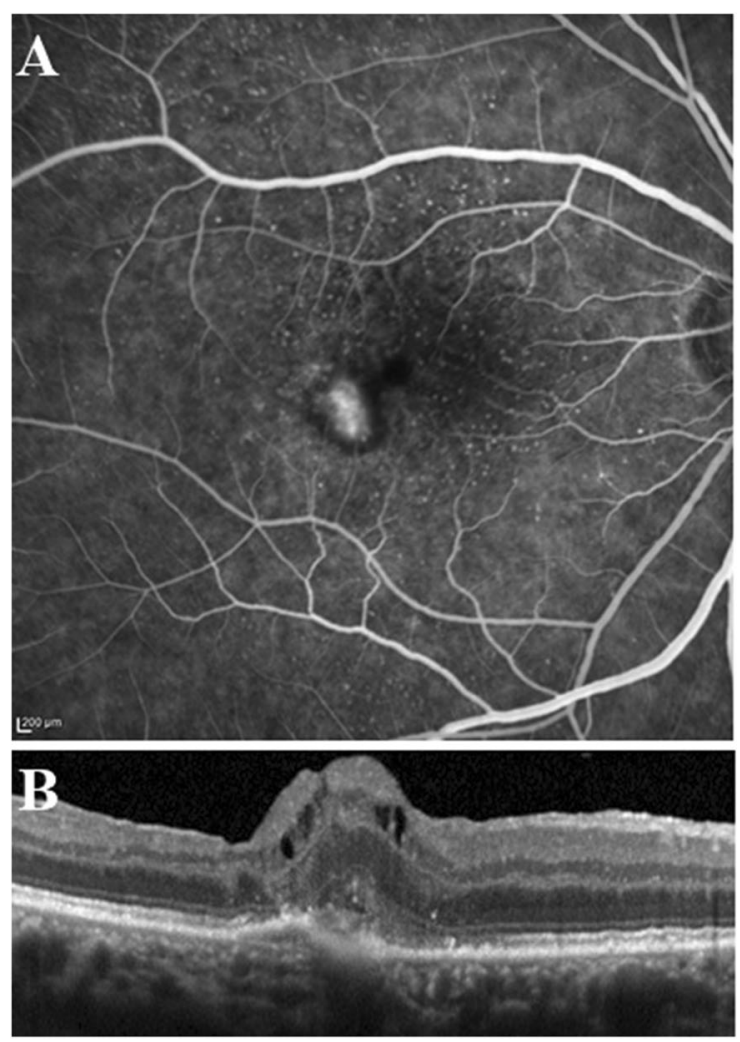

Fig. 4 Well-defined neovascular complex in a 69-year-old female patient who had a history of active mixed-type neovascularization treated with eight anti-VEGF injections 2 years before the optical coherence tomography angiography acquisition. At the presentation, fluorescein angiography image demonstrated a hyperfluorescent area representing the neovascular complex (a). Spectral-domain optical coherence tomography horizontal line scan indicated the neovascular

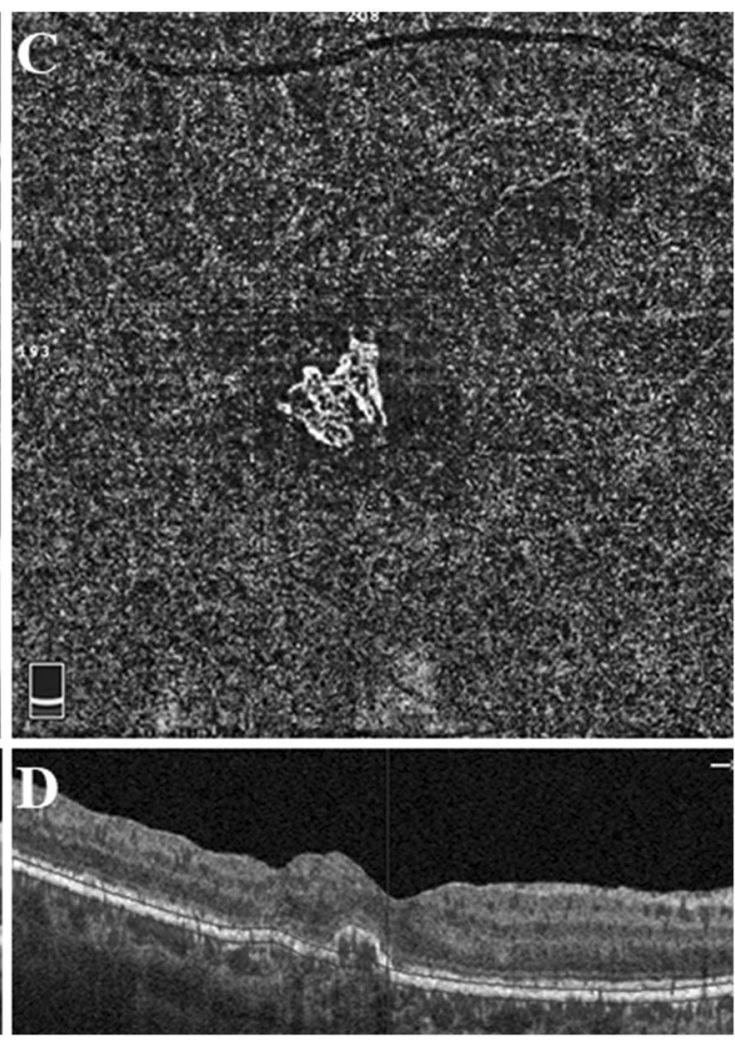

complex both above and beneath the retinal pigment epithelium (b). At the time of study evaluation, the neovascular complex with vessels radiating from one side of the lesion (sea-fan) was demonstrated on the optical coherence tomography angiography en face projection image (c). Cross-sectional optical coherence tomography angiography revealed abnormal flow signal beneath the retinal pigment epithelium in the choroid slabs $(\mathbf{d})$ 
disease inactivity, the detection of a branching smallcapillary network may not reflect disease activity. Also, membrane morphologies were identified more clearly in eyes treated with anti-VEGF than in treatment-naïve eyes, owing to better imaging of the neovascular membrane network after treatment.

\section{Summary}

\section{What was known before}

- Many qualitative features of choroidal neovascularization such as medusa, sea-fan, and pruned-vascular tree have been assessed previously.

- Correlation of clinical activity and the absence or presence of neovascularization on OCTA have also been evaluated.

- It was reported that well-defined vessels tended to be in the clinically active group.

\section{What this study adds}

- If a neovascular network had a long-filamentous appearance, it tended to be clinically inactive.

- Appearance of a dead-tree or pruned-vascular-tree pattern with long dilated filamentous linear vessels on OCTA was associated with chronicity and inactivity of disease.

- But the presence of vessels with a branching smallcapillary network did not always mean clinically active disease.

\section{Compliance with ethical standards}

Conflict of interest The authors declare that they have no conflict of interest.

Ethics approval The study protocol was approved by Institutional Review Board of Sisli Memorial Hospital, Istanbul.

Provenance and peer review Not commissioned; externally peer reviewed.

Publisher's note: Springer Nature remains neutral with regard to jurisdictional claims in published maps and institutional affiliations.

\section{References}

1. Ambati J, Ambati BK, Yoo SH, lanchulev S, Adamis AP. Agerelated macular degeneration: etiology, pathogenesis, and therapeutic strategies. Surv Ophthalmol. 2003;48:257-93.
2. Freund KB, Zweifel SA, Engelbert M. Do we need a new classifiaction for choroidal neovascularization in age-related macular degeneration? Retina. 2010;30:1333-49.

3. Jung JJ, Chen CY, Mrejen S, Gallego-Pinazo R, Xu L, Marsiglia $\mathrm{M}$, et al. The incidence of neovascular subtypes in newly diagnosed neovascular age-related macular degeneration. Am J Ophtalmol. 2014;158:769-79.

4. Querques G, Miere A, Souided EH. Optical coherence tomography angiography of Type 3 neovascularization in age-related macular degeneration. Dev Ophthalmol. 2016;56:57-61.

5. Sulzbacher F, Kiss C, Munk M, Deak G, Sacu S, Schmidt-Erfurth U. Diagnostic evaluation of type 2 (classic) choroidal neovascularization: optical coherence tomography, indocyanine green angiography, and fluorescein angiography. Am J Ophthalmol. 2011;152:799-806 e1.

6. Coscas GJ, Lupidi M, Coscas F, Cagini C, Souied EH. Optical coherence tomography angiography versus traditional multimodal imaging in assessing the activity of exudative age-related macular degeneration. A new diagnostic challenge. Retina. 2015;35: 2219-28.

7. Schmidt-Erfurth U, Chong V, Loewenstein A, Larsen M, Souied E, Schlingemann R, et al. Guidelines for the management of neovascular age-related macular degeneration by the European Society of Retina Specialists (EURETINA). Br J Ophthalmol. 2014;98:1144-67.

8. Kuehlewein L, Bansal M, Lenis TL, Iafe NA, Sadda SR, Bonini Filho MA, et al. Optical coherence tomography angiography of type 1 neovascularization in age-related macular degeneration. Am J Ophthalmol. 2015;160:739-48.

9. Lupidi M, Cerquaglia A, Chhablani J, Fiore T, Singh SR, Cardillo Piccolino F, et al. Optical coherence tomography angiography in age-related macular degeneration: the game changer. Eur J Ophthalmol. 2018;28:349-57.

10. Sulzbacher F, Pollreisz A, Kaider A, Kickinger S, Sacu S, Schmidt-Erfurth U. Identification and clinical role of choroidal neovascularization characteristics based on optical coherence tomography angiography. Acta Ophthalmol. 2017;95:414-20.

11. Miere A, Semoun O, Cohen SY, El Ameen A, Srour M, Jung C, et al. Optical coherence tomography angiography features of subretinal fibrosis in age-related macular degeneration. Retina. 2015;35:2275-84.

12. Al-Sheikh M, Iafe NA, Phasukkijwatana N, Sadda SR, Sarraf D. Biomarkers of neovascular activity in age-related macular degeneration using optical coherence tomography angiography. Retina. 2018;38:220-30.

13. Liang MC, De Carlo TE, Baumal CR, Reichel E, Waheed NK, Duker JS, et al. Correlation of spectral domain optical coherence tomography angiography and clinical activity in neovascular agerelated macular degeneration. Retina. 2016;36:2265-73.

14. Spaide RF. Optical coherence tomography angiography signs of vascular abnormalization with antiangiogenic therapy for choroidal neovascularization. Am J Ophtahlmol. 2015;160:6-16.

15. Lumbroso B, Rispoli M, Savastano C. Longitudinal optical coherence tomography angiography study of type 2 naïve choroidal neovascularization early response after treatment. Retina. 2015;35:2242-51.

16. Xu D, Davila JP, Rahimi M, Rebhun CB, Alibhai AY, Waheed NK, et al. Long-term progression of type 1 neovascularization in age-related macular degeneration using optical coherence tomography angiography. Am J Ophthalmol. 2018;187: 10-20.

17. Miere A, Butori P, Cohen SY, Semoun O, Capuano V, Jung C, et al. Vascular remodeling of choroidal neovascularization after anti-vascular endothelial growth factor therapy visualized on optical coherence tomography angiography. Retina. 2019;39: 548-57. 
18. Iafe NA, Phasukkijwatana N, Sarraf D. Optical coherence tomography angiography of type 1 neovascularization in age-related macular degeneration. Dev Ophthalmol. 2016;56:45-51.

19. Souied EH, El Ameen A, Semoun O, Miere A, Querques G, Cohen SY. Optical coherence tomography angiography of type 2 neovascularization in age-related macular degeneration. Dev Ophthalmol. 2016;56:52-6.

20. Farecki ML, Gutfleisch M, Faatz H, Rothaus K, Heimes B, Spital $\mathrm{G}$, et al. Characteristiscs of type 1 and $2 \mathrm{VNV}$ in exudative AMD in OCT Angiography. Graefes Arch Clin Exp Ophtahlmol. 2017;255:913-21.

21. El Ameen AE, Cohen SY, Semoun O, Miere A, Srour M, Quaranta-El Maftouhi M, et al. Type 2 neovascularization secondary to age-related macular degeneration imaged by optical coherence tomography angiography. Retina. 2015;35:2212-8.

22. Liang MC, Witkin AJ. Optical coherence tomography angiography of mixed neovascularization in age-related macular degeneration. Dev Ophthalmol. 2016;56:62-70. 